\title{
Nitrogen and oxygen photochemistry following SL9
}

\author{
Julianne I. Moses \\ Lunar and Planetary Institute, Houston, Texas \\ Mark Allen \\ Jet Propulsion Laboratory, Pasadena, California \\ G. Randall Gladstone \\ Southwest Research Institute, San Antonio, Texas
}

\begin{abstract}
The collision of SL9 with Jupiter caused many new molecular species to be deposited in the Jovian stratosphere. We use a photochemical model to follow the evolution of the impact-derived species. Our results regarding the nitrogen and oxygen compounds are presented here. $\mathrm{NH}_{3}$ photolysis initiates the nitrogen photochemistry. Much of the nitrogen ends up in $\mathrm{N}_{2}$, nitrogen-sulfur compounds, and $\mathrm{HCN}$, but $\mathrm{NH}_{3}$ and nitriles such as $\mathrm{C}_{2} \mathrm{H}_{3} \mathrm{CN}$ may also exist in observable quantities a year or so after the impacts. Oxygen species survive for a long time in the Jovian stratosphere. The only major oxygen-containing compounds that exhibit dramatic changes in the lower stratosphere in the first year following the impacts are $\mathrm{SO}, \mathrm{SO}_{2}$, and OCS $\mathrm{H}_{2} \mathrm{O}, \mathrm{CO}_{2}$, and $\mathrm{CO}$ are comparatively stable. We discuss the important photochemical processes operating on the nitrogen and oxygen species in the Jovian stratosphere, make predictions concerning the temporal variation of the major species, and identify molecules that might act as good tracers for atmospheric dynamics.
\end{abstract}

\section{Introduction}

Observational results from the Comet $\mathrm{P} /$ ShoemakerLevy 9 (SL9) impacts with Jupiter indicate that cometary debris and tropospheric Jovian air were emplaced in Jupiter's stratosphere. The fact that the emplacement mechanism is complicated and that the molecules continue to evolve due to photolysis, chemical reactions, transport, and condensation makes data analysis difficult. To illuminate some of the complexities of the observations, we have modeled the photochemical evolution of the species that were observed or predicted to have formed immediately following the "splashdown" of an impact plume back into the upper atmosphere. We present the results regarding the nitrogen and oxygen species at this time. Sulfur photochemistry is discussed in another paper [Moses et al., 1995b].

\section{Copyright 1995 by the American Geophysical Union.}

Paper number 95GL01199

0094-8534/95/95GL-01199\$03.00
To minimize any complications due to different physical properties of the comet fragments and to possible nonuniform composition within the plumes, we concentrate our study on the impact sites observed with the Hubble Space Telescope (HST) Faint Object Spectrograph (FOS) [Yelle and McGrath, 1995; Noll et al., 1995]. The HST FOS obtained ultraviolet spectra within the central dark core of the $G$ and $L$ sites during and after the week of the impacts. The signatures of several sulfur and nitrogen species are apparent in these spectra. Other Earth-based observations reveal the presence of OCS, $\mathrm{CO}, \mathrm{H}_{2} \mathrm{O}, \mathrm{SO}_{2}$, and $\mathrm{HCN}$ in the Jovian stratosphere and further confirm the presence of $\mathrm{NH}_{3}$ [e.g., Lellouch et al., 1995; Marten et al., 1994; Bjoraker et al., 1994; Sprague et al., 1995; Meadows et al., 1994; Knacke et al., 1994; Orton et al., 1995; and many others]. However, Noll et al. place upper limits on some key oxygen compounds such as $\mathrm{SO}_{2}, \mathrm{SO}$, and $\mathrm{CO}$ from the HST spectra, and we have assumed that the abundances remain below these upper limits. In a later paper, we will examine the effects of an increase in the abundance of oxygen species on our photochemical model results.

We use a one-dimensional chemical kinetics and diffusion code similar to the one described in Yung et al. [1984] to examine the photochemical evolution of the impact-derived species. Such models are useful in identifying the short- and long-term reservoirs of the different elements, in directing the search for undetected molecular species, and in identifying molecules that might help trace atmospheric dynamics. The predicted temporal variation of the molecular species in our model, when compared with observations, can also teach us about chemical schemes that have not been studied in the laboratory. However, uncertainties in the initial conditions and background atmospheric structure prevent detailed quantitative results from being realized. Our results should be regarded as preliminary and qualitative.

\section{Photochemical Model}

In our photochemical model, which is described in more detail in Moses et al. [1995b], we have tried to identify and include all the important reaction schemes 
that might affect the abundances of the "parent" molecules introduced after the SL9 impacts. Our reaction list is available from the first author upon request. Since we do not yet have enough information to determine how the impacts affected the stratospheric temperature structure and diffusion coefficients, we use parameters relevant to the Northern Equatorial Belt [Gladstone et al., 1995]. Since Zahnle et al. [1995] favor low-pressure shocks for the formation of some of the observed species, we assume that the impact plume splashed back down near 1 mbar, and we deposit most of the impact-derived material above this pressure level. Studies that examine the sensitivity of the model to various initial conditions will be presented at a later date.

We assume that ammonia $\left(\mathrm{NH}_{3}\right)$ has an initial column abundance that is consistent with Noll et al. [1994] but that is an order of magnitude higher than the latest results of Noll et al. [1995] and an order of magnitude lower than the $\mathrm{NH}_{3}$ abundance determined by ground-based infrared observations [Orton et al., 1995; Gierasch et al., 1994]. Hydrogen cyanide (HCN) is observed at submillimeter wavelengths with a reported $\sim 1$ mbar mixing ratio of a few $\times 10^{-6}$ [Owen et al., 1994]; due to a lack of information at the time of our modeling, we arbitrarily assume an initial HCN column abundance that is a factor of six lower than that of $\mathrm{NH}_{3}$ and perhaps an order of magnitude smaller than the observations. Molecular nitrogen $\left(\mathrm{N}_{2}\right)$ is difficult to detect but is likely to be a major nitrogen reservoir following the plume re-entry shock [K. Zahnle and B. Fegley, personal communication, 1994]. We arbitrarily assume that $\mathrm{N}_{2}$ has the same initial abundance as $\mathrm{NH}_{3}$.

$\mathrm{H}_{2} \mathrm{O}, \mathrm{CO}, \mathrm{CO}_{2}, \mathrm{OCS}$, and $\mathrm{SO}_{2}$ have initial column abundances shown in Table 1 . The relative abundances of these oxygen species are in rough agreement with the theoretical predictions of Zahnle et al. [1995] for the case of a plume from a water-rich conet and dry Jovian air splashing back down into the stratosphere; however, we have scaled down the Zahnle et al. numbers to remain below the upper limits given by Noll et al. [1994]. Remember that several observers detected $\mathrm{CO}, \mathrm{OCS}$, and $\mathrm{H}_{2} \mathrm{O}$ at abundances larger than the upper limits proposed by the HST analysis. Since the reason for the discrepancy between the HST and other observations is not known, we concentrate solely on the HST results. Larger assumed abundances of oxygen and nitrogen compounds will change our results; thus, we need to emphasize that our models only pertain tc the small areal regions observed by the HST FOS.

Due to an absence of coherent information at the present, we do not enhance the hydrocarbon abundances after the plume splashdown, but begin with the background hydrocarbon abundances derived by the Gladstone et al. [1995] model for the same $\left(-44^{\circ}\right)$ latitude region. 'The profiles the major hydrocarbon compound are unaffected by the new chemistry.

\section{Results}

The initial nitrogen and oxygen species in our model change with time as photochemical reactions are initiated. Table 1 illustrates the temporal variation of several of the more interesting nitrogen and oxygen compounds. The actual numbers in Table 1 should not be regarded as important since they depend on very uncertain initial conditions, but the relative variation of each species over time should be noted. Column abundances of $\lesssim 10^{10} \mathrm{~cm}^{-2}$ should be considered zero for all practical purposes.

\section{Nitrogen Photochemistry}

The kinetics of nitrogen compounds in a reducing atmosphere are fairly well studied, and rates for most of the nitrogen reactions in our model are available in the literature. The nitrogen photochemistry is initiated by the photolysis of $\mathrm{NH}_{3}$ in the $170-220 \mathrm{~nm}$ region. The photolysis lifetime for ammonia at $-44^{\circ}$ latitude in our diurnally averaged model is 5 days at $10^{-5}$ mbar - a time scale considerably longer than that for the pho-

Table 1. Time Variation of Column Abundances $\left(\mathrm{cm}^{-2}\right)$

\begin{tabular}{lcccccc}
\hline Species & Initial & 9 hrs & 3 days & 9 days & 30 days & 17 months \\
\hline $\mathrm{N}_{2}$ & $1.5 \times 10^{17}$ & $1.5 \times 10^{17}$ & $1.5 \times 10^{17}$ & $1.5 \times 10^{17}$ & $1.6 \times 10^{17}$ & $1.8 \times 10^{17}$ \\
$\mathrm{NH}_{3}$ & $1.5 \times 10^{17}$ & $1.5 \times 10^{17}$ & $1.2 \times 10^{17}$ & $7.2 \times 10^{16}$ & $4.1 \times 10^{16}$ & $3.7 \times 10^{14}$ \\
$\mathrm{~N}_{2} \mathrm{H}_{4}$ & - & $2.0 \times 10^{13}$ & $3.5 \times 10^{13}$ & $1.4 \times 10^{14}$ & $2.0 \times 10^{14}$ & $2.2 \times 10^{10}$ \\
$\mathrm{CH}_{2} \mathrm{NH}$ & - & $2.7 \times 10^{10}$ & $1.0 \times 10^{11}$ & $6.8 \times 10^{12}$ & $6.1 \times 10^{13}$ & $2.8 \times 10^{12}$ \\
$\mathrm{CH}_{3} \mathrm{NH}_{2}$ & - & $4.8 \times 10^{10}$ & $1.5 \times 10^{11}$ & $3.9 \times 10^{13}$ & $1.4 \times 10^{14}$ & $7.0 \times 10^{10}$ \\
$\mathrm{HCN}$ & $2.4 \times 10^{16}$ & $2.4 \times 10^{16}$ & $2.4 \times 10^{16}$ & $2.4 \times 10^{16}$ & $2.4 \times 10^{16}$ & $2.7 \times 10^{16}$ \\
$\mathrm{HC} \mathrm{N}$ & - & $1.8 \times 10^{10}$ & $1.3 \times 10^{11}$ & $2.9 \times 10^{11}$ & $5.8 \times 10^{11}$ & $5.8 \times 10^{11}$ \\
$\mathrm{C}_{2} \mathrm{H}_{3} \mathrm{CN}$ & - & $2.3 \times 10^{11}$ & $3.3 \times 10^{12}$ & $2.3 \times 10^{13}$ & $1.3 \times 10^{14}$ & $1.1 \times 10^{15}$ \\
$\mathrm{NS}$ & - & $3.3 \times 10^{15}$ & $3.0 \times 10^{16}$ & $5.9 \times 10^{16}$ & $6.8 \times 10^{16}$ & $6.1 \times 10^{16}$ \\
$\mathrm{HNCS}$ & - & $7.0 \times 10^{14}$ & $7.7 \times 10^{15}$ & $2.0 \times 10^{16}$ & $2.7 \times 10^{16}$ & $3.2 \times 10^{16}$ \\
$\mathrm{H}_{2} \mathrm{O}$ & $1.8 \times 10^{14}$ & $1.8 \times 10^{14}$ & $1.8 \times 10^{14}$ & $1.8 \times 10^{14}$ & $1.8 \times 10^{14}$ & $1.7 \times 10^{14}$ \\
$\mathrm{CO}$ & $1.8 \times 10^{14}$ & $1.8 \times 10^{14}$ & $1.8 \times 10^{14}$ & $1.8 \times 10^{14}$ & $1.9 \times 10^{14}$ & $2.1 \times 10^{14}$ \\
$\mathrm{CO}$ & $3.0 \times 10^{12}$ & $3.0 \times 10^{12}$ & $3.0 \times 10^{12}$ & $3.0 \times 10^{12}$ & $3.0 \times 10^{12}$ & $3.0 \times 10^{12}$ \\
$\mathrm{SO}$ & - & $1.4 \times 10^{12}$ & $8.8 \times 10^{12}$ & $1.0 \times 10^{13}$ & $4.0 \times 10^{12}$ & $5.0 \times 10^{10}$ \\
$\mathrm{SO}_{2}$ & $1.1 \times 10^{13}$ & $1.0 \times 10^{13}$ & $6.2 \times 10^{12}$ & $2.0 \times 10^{12}$ & $2.6 \times 10^{10}$ & $1.5 \times 10^{6}$ \\
$\mathrm{OCS}$ & $3.0 \times 10^{12}$ & $3.0 \times 10^{12}$ & $2.7 \times 10^{12}$ & $2.2 \times 10^{12}$ & $1.1 \times 10^{12}$ & $2.8 \times 10^{11}$ \\
\hline
\end{tabular}

Times refer to approximate times after plume splashdown. 
tochemically active sulfur species. Fig. 1 shows the time variation of $\mathrm{NH}_{3}$ following the impacts. The primary pathway for ammonia photolysis on Jupiter is $\mathrm{NH}_{3}+h \nu \rightarrow \mathrm{NH}_{2}+\mathrm{H}$. In a mechanism never before encountered in the atmospheres of the planets, the $\mathrm{NH}_{2}$ radicals formed during this process may react with the copious amounts of sulfur and organo-sulfur radicals present after the impacts; however, very little is known about sulfur-nitrogen vapor-phase chemistry. In our model, NS, HNS, and isothiocyanic acid (HNCS) comprise our representative $\mathrm{S}-\mathrm{N}$ species; we find that large quantities of NS and HNCS are formed within the first days or so after the impacts (see Table 1). The formation mechanisms for these compounds are described in Moses et al. [1995b]. We have omitted photolysis as a loss mechanism for these species even though they all absorb near-ultraviolet radiation. Other possible important $\mathrm{S}-\mathrm{N}$ species that are not included in the model are $\mathrm{SCN}$; $\mathrm{HSCN} ; \mathrm{CH}_{3} \mathrm{SCN} ; \mathrm{N}_{\mathrm{x}} \mathrm{S}_{\mathrm{y}}$ radicals, rings, or polymers; $\mathrm{S}_{x} \mathrm{NH}$; $\mathrm{NCS} ; \mathrm{CH}_{3} \mathrm{NCS}$; and other isothiocyanates. Although our predictions regarding NS and HNCS are highly speculative, positive or negative searches for these molecules at UV, infrared, and longer wavelengths would be useful in helping to resolve several of the proposed photochemical schemes.

If the $\mathrm{NH}_{2}$ radicals produced by $\mathrm{NH}_{3}$ photolysis do not react effectively with sulfur compounds, then the most likely fate of the $\mathrm{NH}_{2}$ would be to recycle ammonia; in that case, hydrazine $\left(\mathrm{N}_{2} \mathrm{H}_{4}\right)$ would also form in significant quantities. Observations of the rate of disappearance of $\mathrm{NH}_{3}$ following the SL9 impacts should shed further light on this issue. If the $\mathrm{NH}_{3}$ abundance remains relatively constant over the course of a week or so, then $\mathrm{NH}_{2}$ reactions with sulfur radicals are probably not active loss pathways, and nitrogen-sulfur species will not be significant reservoirs for the nitrogen. Groundbased infrared observations yet to be published should be sufficiently detailed as to provide this information. Preliminary analyses of the HST data [e.g., Yelle and McGrath, 1995] suggest that the $\mathrm{NH}_{3}$ lifetime in our model is consistent with the data.

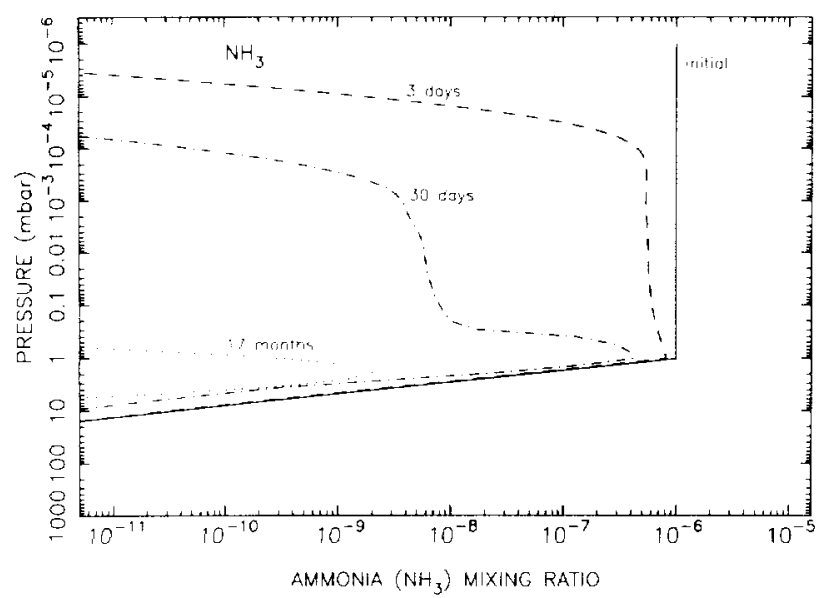

Figure 1. The evolution of $\mathrm{NH}_{3}$. 'The solid line represents the initial abundance in our model, the dashed line is for 3 days after the impacts, the dot-dashed line is for 30 days after the impact, and the dotted line is for 17 months after the impact.
Hydrogen cyanide may be produced in large quantities during the plume splashdown $[K$. Zahnle and $B$. Fegley, personal communication, 1995]. Although HCN does not absorb in the near ultraviolet, it is dissociated by photons of wavelength less than $190 \mathrm{~nm}$. Its lifetime against photolysis is 17 days at $10^{-5}$ mbar and much longer in the lower stratosphere. Thus, very little change in the HCN abundance is observed in our model over the course of a year. HCN might therefore be regarded as a good indicator of other changes in the stratosphere - any changes in the HCN observations would be due to temperature variations or horizontal spreading rather than to changes in total abundance.

Molecular nitrogen $\left(\mathrm{N}_{2}\right)$ has no efficient photochemical loss mechanisms in the Jovian stratosphere. In our model, the $\mathrm{N}_{2}$ abundance increases slightly with time due to reactions such as $\mathrm{N}+\mathrm{NS} \rightarrow \mathrm{N}_{2}+\mathrm{S}$ where the atomic $\mathrm{N}$ is generated by reactions of $\mathrm{NH}$ with $\mathrm{H}$ and hydrocarbon radicals, and the atomic $S$ is generated by $\mathrm{S}_{2}$ photolysis. Moderate abundances of nitriles such as acrylonitrile $\left(\mathrm{C}_{2} \mathrm{H}_{3} \mathrm{CN}\right)$ are observed in our model after several months. Searches for these molecules may be useful in identifying important photochemical reaction mechanisms.

Since we have assumed that the impact-derived species are deposited at altitudes above 1 mbar in our model, the nitrogen-bearing molecules are not abundant enough in the lower stratosphere to become supersaturated. Therefore, they do not condense. If these species do extend throughout the lower stratosphere, then several, especially the nitriles, may be condensable.

\section{Oxygen Photochemistry}

Although Earth-based observers identified oxygencontaining compounds at some of the impact sites, no oxygen compounds (with the possible exception of $\mathrm{H}_{2} \mathrm{O}$ ) were identified in the HST FOS spectra. Therefore, we limited the initial abundances of oxygen compounds in our model, and oxygen species do not play a major role in the photochemical evolution of the impact regions. Water photolyzes at relatively short wavelengths (140

$190 \mathrm{~nm}$ ), so its lifetime against photolysis is long (38 days at $10^{-5}$ mbar). The primary photolysis pathway is the production of $\mathrm{H}$ and $\mathrm{OH}$, but some $\mathrm{O}\left({ }^{1} \mathrm{D}\right)$ formation is also possible below $177 \mathrm{~nm}$. The $\mathrm{OH}$ radicals either react with the $\mathrm{S}$ atoms to form $\mathrm{SO}$ in the middle stratosphere or recycle $\mathrm{H}_{2} \mathrm{O}$ at other altitudes.

The $\mathrm{SO}$ abundance increases at first due to the above reaction and to the photolysis of $\mathrm{SO}_{2}$ (with a photolysis lifetime of 2.8 days at $10^{-5} \mathrm{mbar}$, but $\mathrm{SO}$ itself is photolyzed in the $190-230 \mathrm{~nm}$ region (lifetime 1.5 days) and is eventually lost from the stratosphere. The ultimate fate of most of the oxygen is the production of $\mathrm{CO}$, which is very stable in the Jovian stratosphere. $\mathrm{CO}$ is produced directly by the photolysis of $\mathrm{CO}_{2}$ and $\mathrm{OCS}$ and indirectly by reactions such as $\mathrm{S}+\mathrm{OCS} \rightarrow \mathrm{CO}+\mathrm{S}_{2}$ and $\mathrm{O}+\mathrm{OCS} \rightarrow \mathrm{CO}+\mathrm{SO}$. Minor amounts of formaldehyde $\left(\mathrm{H}_{2} \mathrm{CO}\right)$, ketene $\left(\mathrm{H}_{2} \mathrm{CCO}\right)$, and acetaldehyde $\left(\mathrm{CH}_{3} \mathrm{CHO}\right)$ form in the first weeks and months after the impact, but the low initial column abundance of oxygen compounds in our model precludes 
these species from forming in appreciable quantities. If oxygen species are indeed more abundant as a whole than is indicated by the spatially limited HST FOS spectral sample, then these conclusions would change. Note that the much larger reported abundances of $\mathrm{H}_{2} \mathrm{O}$, CO, and OCS reported by Bjoraker et al. [1994], Knacke et al. [1994], Lellouch et al. [1995], and Marten et al. [1994] suggest that oxygen compounds may be more important components at the impact sites than we have assumed.

The stability of $\mathrm{CO}$ in the Jovian stratosphere might make it a good tracer for atmospheric dynamics; however, the peak of the mixing ratio profile for $\mathrm{CO}$ migrates downward markedly over the first year due to diffusion and to the progressive loss of short-lived parent species [see Fig. 3 of Moses et al., 1995a]. Such vertical variations might confuse observational analyses. This result further illustrates the usefulness of photochemical models.

\section{Summary and Conclusions}

The nitrogen and oxygen species introduced to the Jovian stratosphere following the SL9 impacts are fairly long-lived. Within the first several days and weeks, oxygen and nitrogen photolysis products may react with the abundant sulfur and $\mathrm{SH}$ radicals present at the impact sites. The photochemical schemes that we adopt for the nitrogen-sulfur reactions have never been studied in laboratories here on Earth; thus, the impacts may provide us with a unique look at some unusual atmospheric photochemistry. In our model, the primary reservoirs for the nitrogen species after $\sim 1$ year are $\mathrm{N}_{2}, \mathrm{HCN}$, and sulfur-nitrogen species such as NS and HNCS. Nitriles such as $\mathrm{CH}_{3} \mathrm{CN}, \mathrm{HC}_{3} \mathrm{~N}$, and $\mathrm{C}_{2} \mathrm{H}_{3} \mathrm{CN}$ may also form in observable quantities. Our low initial assumed abundances for the oxygen compounds preclude oxygen from playing a major role in the stratospheric photochemistry. $\mathrm{H}_{2} \mathrm{O}, \mathrm{CO}$, and $\mathrm{CO}_{2}$ are all fairly stable in the Jovian stratosphere. $\mathrm{H}_{2} \mathrm{O}$ will eventually be converted to $\mathrm{CO}$, with a smaller transient production of $\mathrm{SO}, \mathrm{SO}_{2}, \mathrm{H}_{2} \mathrm{CO}, \mathrm{CH}_{3} \mathrm{OH}, \mathrm{H}_{2} \mathrm{CCO}$, and $\mathrm{CH}_{3} \mathrm{CHO}$. Nitrogen-oxygen species such as $\mathrm{NO}$ and $\mathrm{NO}_{2}$ are unimportant in our model. $\mathrm{HCN}$ and $\mathrm{CO}$, both produced readily during the plume splashdown and both observable at millimeter and submillimeter wavelengths, might be good tracers for atmospheric dynamics, but caution should be exerted when applying this suggestion. CO and other seemingly stable oxygen compounds exhibit much structure in their vertical profiles even though their total column abundances remain nearly constant. Our predictions concerning the temporal variation of important oxygen and nitrogen compounds are given in Table 1 . All the results in this paper depend on the uncertain initial conditions and reaction rates that we have adopted in our model - our conclusions should be regarded as highly speculative.

Acknowledgments. We are grateful to K. Zahnle and B. Fegley for useful discussions. This work was supported by the Lunar and Planetary Institute and represents LPI contribution number 859. Support from NASA grant NAGW 1509 and NSF grant ATM-9322506 is also gratefully acknowledged.

\section{References}

Bjoraker, G. L., et al., Detection of water in the fireball of fragments $\mathrm{G}$ and $\mathrm{K}$ of Comet Shoemaker-Levy 9 , Bull. Am. Astron. Soc., 26, 1578-1589, 1994.

Gierasch, P., et al., A physical interpretation of the SL-9 impacts observed from Palomar, Bull. Am. Astron. Soc., 26, 1585, 1994.

Gladstone, G. R., M. Allen, and Y. L. Yung, Hydrocarbon photochemistry in the upper atmosphere of Jupiter, manuscript in preparation, 1995.

Knacke, R. F., T. R. Geballe, K. S. Noll, and T. Y. Brooke, Infrared spectra of the $\mathrm{R}$ post-impact events of Comet Shoemaker-Levy 9, Bull. Am. Astron. Soc., 26, 1589, 1994.

Lellouch, E., et al., Chemical and thermal response of Jupiter's atmosphere following the impact of comet. Shoemaker-Levy 9, Nature, 373, 592-595, 1995.

Marten, A., et al., New heterodyne millimeter observations of Jupiter performed after the collision of comet SL9 with the planet, Bull. Am. Astron. Soc., 26, 1589-1590, 1994.

Meadows, V., et al., AAT observations of Shoemaker Levy-9 collisions with Jupiter, Bull. Am. Astron. Soc., 26, 1588, 1994.

Moses, J. I., M. Allen, and G. R. Gladstone, Nitrogen and oxygen photochemistry at the Shoemaker-Levy 9 impact sites, Lunar Planet. Sci., XXVI, 999-1000, 1995a.

Moses, J. I., M. Allen, and G. R. Gladstone, Post-SL9 sulfur photochemistry on Jupiter, Geophys. Res. Lett., This issue, 1995b.

Noll, K. S., et al., Hubble Space Telescope spectroscopic observations of Jupiter after the impact of Comet SL9, Bull. Am. Astron. Soc., 26, 1576-1577, 1994.

Noll, K. S., et al., HST spectroscopic observations of Jupiter after the collision of Comet Shoemaker-Levy 9, Science, $267,1307-1313,1995$.

Orton, G., et al., Collision of Comet Shoemaker-Levy 9 with Jupiter observed by the NASA Infrared Telescope Facility, Science, 267, 1277-1281, 1995.

Owen, T., et al., JCMT Observations of the collision of Comet Shoemaker-Levy 9 with Jupiter, Bull. Am. Astron. Soc., 26, 1584, 1994.

Sprague, A. L., et al., Sulfur dioxide following the impacts of the SL-9 R and W fragments, Submitted to Geophys. Res. Lett., 1995.

Yelle, R. V., and M. A. McGrath, Ultraviolet spectroscopy of the SL9 impact sites, I: The $175-230 \mathrm{~nm}$ region, Submitted to Icarus, 1995.

Yung, Y. L., M. Allen, and J. P. Pinto, Photochemistry of the atmosphere of Titan: Comparison between model and observations, Astrophys. J.Suppl. Ser., 55, 465-506, 1984.

Zahnle, K., M.-M. MacLow, K. Lodders, and B. F. Fegley, Jr., Sulfur chemistry in the wake of Comet ShoemakerLevy 9, Geophys. Res. Lett., In press, 1995.

M. Allen, JPL 183-601, Pasadena, CA 91109.

G. R. Gladstone, Southwest Research Institute, PO

Drawer 28510, 6220 Culebra Rd., San Antonio, TX 78284

J. I. Moses, Lunar and Planetary Institute, 3600 Bay Area Blvd., Houston, TX 77058
(Received December 29, 1994; revised March 10, 1995; accepted March 24, 1995.) 